Revue d'histoire de l'Amérique française

PBS REVUE D.HISTOIRE DE L'AMÉRIQUE FRANÇAISE

\title{
La Canadian Seamen's Union (1936-1949) : un chapitre de l'histoire du mouvement ouvrier canadien
}

\section{Robert Comeau}

Volume 29, numéro 4, mars 1976

URI : https://id.erudit.org/iderudit/303483ar

DOI : https://doi.org/10.7202/303483ar

Aller au sommaire du numéro

Éditeur(s)

Institut d'histoire de l'Amérique française

ISSN

0035-2357 (imprimé)

1492-1383 (numérique)

Découvrir la revue

Citer cet article

Comeau, R. (1976). La Canadian Seamen's Union (1936-1949) : un chapitre de l'histoire du mouvement ouvrier canadien. Revue d'histoire de l'Amérique française, 29(4), 503-538. https://doi.org/10.7202/303483ar d'utilisation que vous pouvez consulter en ligne. 


\title{
LA CANADIAN SEAMEN'S UNION (1936-1949): UN CHAPITRE DE L'HISTOIRE DU MOUVEMENT OUVRIER CANADIEN *
}

\author{
ROBERT COMEAU \\ Département d'bistoire \\ Université du Québec à Montréal \\ À Norman Bethune
}

L'histoire des marins canadiens organisés dans la Canadian Seamen's Union (1936-1949)** constitue un chapitre significatif de l'histoire du mouvement ouvrier canadien.

La CSU est un exemple de syndicat militant qui a mené des luttes particulièrement vives pour améliorer les conditions de travail et les conditions salariales des travailleurs maritimes.

Le rôle joué par les communistes à l'intérieur de ce syndicat illustre la position du Parti communiste canadien (PCC) sur la question syndicale, position qui sera largement redéfinie à la suite du $7^{\mathrm{e}}$ congrès de l'Internationale communiste (mars 1935) qui marque un tournant dans l'histoire du PCC.

\footnotetext{
Sigles employés:

American Federation of Labour (AFL) - Canadian Fishermen's and Fishlander's Union (CFFU) - Canadian Lake Seamen's Union (CLSU) - Canadian Seamen's Union (CSU) - Congrès canadien du Travail (CCT) - Congrès des métiers et du travail du Canada (CMTC) - Congress of Industrial Organization (CIO) - Internationale communiste (IC) - International Seamen's Union (ISU) - National Maritime Union (NMU)

* L'auteur tient à remercier M. Harry Davis sans lequel ce travail n'aurait pas été rendu possible ainsi que MM. Stanley Bréhaut Ryerson, Léo Roback et Charles Lipton. L'auteur assume l'entière responsabilité de son texte qui n'engage aucunement celle du groupe de recherches sur le mouvement ouvrier politique (MOP) de l'UQAM. L'auteur remercie également MM. B. Dansereau, P.-A. Linteau et J. Samson qui lui ont fait part de leurs critiques.

** Nous utiliserons également l'appellation française «Union des marins canadiens" moins employée à l'époque.
} 
C'est grâce au journal produit par le syndicat des marins (CSU), le Searchlight, que nous avons pu retracer leurs conditions concrètes de travail, connaître leurs principales revendications, et suivre les luttes qu'ils ont menées, particulièrement lors des grandes grèves de 1946 et 1949. Le Searchlight *** est un instrument particulièrement précieux à cet égard. Principal, parfois unique moyen d'échanges entre les équipages et les officiers de l'Union, il servait à la fois à informer les marins sur le travail des permanents et les développements du syndicat, à assurer la démocratie dans l'organisation syndicale et à remplacer les assemblées durant la saison de navigation.

Chaque comité de navire où se trouvaient des syndiqués à la CSU correspondait régulièrement avec les divers bureaux du syndicat pour faire valoir ses griefs. On retrouve donc dans ce journal non seulement les ententes officielles conclues entre le comité de négociations de la CSU et les armateurs, des analyses des rapports de forces entre les propriétaires des compagnies et les marins, des informations proprement syndicales, mais aussi les nombreuses lettres des marins qui dénoncent leurs conditions de travail et l'attitude des «capitaines» à bord. La condition particulière de ces travailleurs itinérants a favorisé l'expression écrite de centaines de témoignages de travailleurs exploités. Le journal permet de rendre compte également des rapports entre la base et la direction. À ce sujet, l'attitude de la direction communiste de la CSU nous est apparue exemplaire. Non seulement, encourage-t-elle la libre discussion à la base mais elle ne freine pas le militantisme des marins: la direction de la CSU ne craint pas d'affronter la direction du Congrès des métiers et du travail du Canada (CMTC) pour défendre les positions de la CSU adoptées lors de ses assemblées générales.

Même si l'on a pu déceler des tendances économistes dans la propagande menée par la direction communiste de la CSU, on peut constater que les thèmes de cette propagande ne se limitent pas aux revendications économiques des marins; on n'ignore pas leur portée politique. L'importance des dénonciations du fascisme, de l'anticommunisme, et de l'internationalisme prolétarien ainsi que la prise en considération de l'ensemble du mouvement ouvrier sont significatifs à cet égard.

\footnotetext{
*** Vol. 1, no 1 (ler juillet 1937) au vol. XII. no 18 (24 décembre 1948). Le bimensuel devienit bilingue, format tabloïd imprimé, le 24 août 1940 (vol IV, no 8). Une analyse systématique de ce journal est en cours.
} 
La direction communiste de la CSU prévenant les tentatives de la bourgeoisie et de ses alliés, pour isoler l'avant-garde plus consciente des autres travailleurs, réussira à cimenter cette unité indispensable. Le Searchlight fait largement écho à toutes les luttes ouvrières importantes, surtout après la guerre et travaille à la promotion de l'unité ouvrière et à la lutte anticapitaliste. Après cinq mois d'existence, il subissait déjà les foudres du pouvoir bourgeois: le 10 novembre 1937, la loi du cadenas frappait ce journal. Un numéro spécial miméographié (vol I, no 10) explique que l'imprimerie où se trouvait tout l'équipement a été cadenassée. Et dès le 15 novembre 1937, la CSU réussissait à publier dans son format régulier.

Nous avons retracé les grandes étapes de l'évolution de la CSU et de ses rapports avec les armateurs, les gouvernements canadiens et québécois et la direction du CMTC. C'est ainsi que le changement de tactique du PCC face à la guerre a des conséquences sur le travail syndical. La répression contre la CSU en 1940-41 n'est pas étrangère au fait que durant ces années, le PCC dénonce la guerre impérialiste et la menace de conscription, alors qu'à partir de 1942, la tactique de collaboration avec le gouvernement dans l'effort de guerre contre le fascisme se retrouve au niveau syndical. Dans l'immédiat après-guerre, nous verrons dans le contexte de la guerre froide, les efforts conjugués du patronat et du parti libéral en vue d'évincer et le parti communiste et ce syndicat combatif, en utilisant les dirigeants corrompus d'un syndicat international rival. C'est l'épisode tragique de la grève déclenchée le 31 mars 1949 qui marque la destruction de la CSU. En même temps, le PCC ne réussira plus à maintenir la combativité qui l'animait jusque-là.

Ce travail a ses limites: il ne faudrait pas y voir une étude systématique des activités du Parti communiste canadien ou une étude globale de l'activité syndicale communiste. L'enquête sur le PCC doit se poursuivre. Elle est indispensable à tous ceux qui revendiquent l'héritage des communistes canadiens. Nous avons beaucoup à apprendre de l'histoire de ces milliers d'obscurs militants et de ses dirigeants. Pour l'instant rappelons les grandes lignes de l'intervention du PCC dans le domaine syndical.

Le septième congrès de l'Internationale communiste (IC), en juillet 1935, qui sanctionne la tactique du «front uni», marque un changement important dans le travail des militants communistes canadiens à l'intérieur des syndicats. Le Parti communiste canadien, devant la nécessité de bloquer le processus de fascisation, revise sa 
stratégie. La Ligue d'unité ouvrière, centrale syndicale communiste (1929-1935), est dissoute, conformément aux directives de l'IC d'abolir les syndicats «rouges» et de rejoindre les rangs du mouvement syndical le plus large. Cette liquidation de l'organisation de lutte syndicale ne se fit pas sans difficulté ${ }^{1}$. On invita les anciens membres de la Ligue d'unité ouvrière à rejoindre les rangs des syndicats internationaux affiliés au Congrès des métiers et du travail du Canada (CMTC) ou après 1936, à devenir organisateurs pour le Congress of Industrial Organization (CIO), lequel demeura affilié au CMTC jusqu'en 1939. Au Canada, la majorité des organisateurs du CIO étaient des communistes ${ }^{2}$ ou sympathisants ayant acquis leur expérience à la Ligue d'unité ouvrière ${ }^{3}$.

Les militants communistes mènent durant ces années un travail d'organisation syndicale exemplaire, si bien qu'en 1937 ils contrôlent près du tiers des syndicats canadiens affiliés à la CIO, soit plus de 150,000 travailleurs, C'est l'époque de la fondation des grands syndicats industriels puissants: le Syndicat industriel des ouvriers de I'automobile, le comite d'organisation des metalílurgistes (Ies futurs Métallurgistes unis d'Amérique), le syndicat des ouvriers du caoutchouc et des produits électriques en Ontario, le syndicat des marins des Grands lacs, celui des ouvriers de la forêt et des ports de la Colombie britannique et celui des mineurs de l'Ouest. De nombreux communistes militent dans ces syndicats. Au Québec, la pénétration du syndicalisme industriel est moins rapide et plus limitée selon An-

1 Le groupe de militants de la revue Mobilisation présente ainsi cette décision de la direction du PCC: «Mécaniquement, les communistes canadiens en viennent à conclure qu'il faut rejoindre les rangs de l'AFL. De plus, plusieurs erreurs furent commises dans le processus de dissolution de la Ligue. Ainsi à plusieurs endroits, des directives sont avancées par en haut, sans explication et discussion à la base, ce qui amènera plusieurs militants de longue date à quitter le Parti parce qu'ils ne comprenaient pas la décision de dissoudre l'organisation de lutte qu'ils avaient contribué à bâtir dans leur sueur et dans leur sang." Mobilisation, 4, no 4, chapitre 3, «Le Front contre le Fascisme 1935-1945»: 13.

2 Voir I.M. Abella, "The CIO, the Communist Party and the formation of the Canadian Congress of Labor, 1936-1941», Canadian Historical Association, Historical Papers, 1969: 112-118.

3 «Au Canada, les syndicats industriels du CIO se développent à plusieurs endroits sur la base des anciens syndicats de la Ligue d'unité ouvrière. Ce fait est d'ailleurs encouragé par la direction du CIO, qui a choisi comme politique d'utiliser au maximum les possibilités d'organisation et d'agitation des communistes qui, trop contents de faire enfin partie du mouvement syndical officiel, embarquent dans cette campagne avec toutes leurs énergies». Mobilisation, 4, no 4: 13. 
drée Lévesque-Olssen, elle se limite aux secteurs suivants: métallurgie, abattoirs, charbon et cuir ${ }^{4}$.

La répression contre les syndicats CIO par Duplessis et le clergé réactionnaire va forcer également certains communistes à travailler à la base dans les syndicats catholiques, en particulier dans l'industrie du textile-coton.

Durant cette période de «front uni», le PCC favorisait l'affiliation du CIO au CMTC. Mais quand en 1939, sous la pression de l'American Federation of Labour (AFL) le CMTC a exclu les syndicats $\mathrm{CIO}$, les communistes ont pu prendre le contrôle du Comité canadien du CIO jusqu'en septembre 1940, date de la fondation du Congrès canadien du Travail (1940-1956). Le CCT contrairement au CMTC favorisera les unions industrielles et s'efforcera de garder un caractère plus « canadien».

Même si de nombreux syndicats affiliés de l'AFL se détachent de cette centrale pour s'affilier à la nouvelle CIO, la presse communiste met de l'avant le thème de l'unité ouvrière. Clarté, organe du PCC au Québec de 1935 à 1939, ne s'intéresse pas qu'à l'organisation CIO. Le PCC incite aussi les travailleurs à joindre les rangs des syndicats modérés du CMTC et à mettre en sourdine les vieilles rivalités intersyndicales. Des militants communistes vont même occuper des postes de direction au sein du Congrès des métiers et du travail. Le meilleur exemple est J. A. Pat Sullivan, présidentfondateur de la CSU, qui, en septembre 1937, sera élu avec Sidney Sarkin à l'exécutif du Conseil de Montréal du CMTC; Sullivan sera vice-président du CMTC en $1942^{5}$.

Andrée Lévesque-Olssen explique la situation ainsi:

His success in the TLC accordent well with current communist objectives and was made easier by the TLC's avowed aloofness from political parties. Had the TLC committed itself to a political party such as the CCF, a communist could not have been tolerated in such a powerful position. As it was, the overt political stand of the international unions made it fairly easy for communists to join. ${ }^{6}$

4 Andrée Lévesque-Olssen. The Canadian Left in Quebec during the Great Depression: The Communist Party of Canada and the Co-operative Commonwealth Federation in Quebec 1929-1939. Thèse de Doctorat (Duke University, 1972), 150. 1947.

5 Pat Sullivan sera élu secrétaire-trésorier en 1943 jusqu'à sa démission en

6 André Lévesque-Olssen, op.cit., 152-153. 
Ce qui ne veut pas dire qu'à cette époque le CMTC était toujours procommuniste; mais le fait qu'il ouvrait ses portes à tous sans considération des affiliations politiques profita aux communistes durant cette période.

\section{I - 1936-1940: campagne de syndicalisation}

Les premières tentatives pour améliorer les conditions des travailleurs maritimes ont été entreprises par des militants de la Ligue d'unité ouvrière. Le premier syndicat de marins canadiens a été fondé vers 1934 par Dewar Ferguson, un membre actif du Parti communiste du Canada à Toronto.

À Montréal, avant la fondation de la Canadian Seamen's Union (CSU), il n'y avait qu'une association de boutique, la National Seamen's Association, dirigée par le capitaine McMasters; elle regroupait 600 membres. Pat Sullivan, président-fondateur du CSU, la décrit en ces termes:

Captain MícḾasters (...) ran the Union as a fañily âffäir, with himself as President and his daughter as Secretary-Treasurer. There were no elections and the seamen had no voice in the running of the organization. ${ }^{7}$

Les armateurs tenteront de maintenir par tous les moyens cette «association» jusqu'en 1938, multipliant les intimidations envers les marins pour qu'ils réintègrent cette association.

D. Ferguson dirigeait le syndicat Marine Workers Union of the Great Lakes lorsque Pat Sullivan et quelques stewards entrèrent en contact avec des membres du PCC à Montréal. La charte qui fut accordée à ce nouveau syndicat fondé par Sullivan était émise en 1936 au nom de National Seamen's Union affilié au Congrès pan-canadien du Travail. À l'été 1936, le syndicat de Sullivan qui regroupait 320 membres se fusionnait au syndicat dirigé par Ferguson. Ce dernier ne regroupait qu'une cinquantaine de militants. La fusion réalisée, on conserva le nom National Seamen's Union avant d'adopter un peu plus tard le nom définitif de Canadian Seamen's Union ${ }^{8}$. Ferguson travaillait à l'organisation à Toronto, Sullivan à Montréal. Le premier bureau de la CSU fut ouvert à Montréal en avril 1936 et le

7 Pat Sullivan, Red Sails on the Great Lakes (Toronto, MacMillan, 1955), 11. Les 3 premiers chapitres relatent les débuts du syndicalisme maritime.

8 Tim Buck décrit la fondation du CSU, dans son histoire du Parti communiste canadien (1922-1952), in 30 Years (Toronto, Progress Books, 1952), 119. 
deuxième à Toronto en juillet. A la fin de l'année 1936 la CSU avait rallié dans ses rangs 1,200 marins ${ }^{9}$.

Lorsqu'il fut question d'affiliation à une centrale internationale, deux possibilités s'offraient: une affiliation avec l'International Seamen's Union (All Canadian Congress Labor) ou avec la National Maritime Union (NMU). La première (ISU) était le syndicat institué sur la côte est des États-Unis peu de temps après la première guerre mondiale. Ce syndicat déclina pendant les années trente, alors que la Sailors Union of the Pacific prospérait. L'AFL prit le contrôle de l'ISU, élimina les communistes qui y avaient exercé initialement la direction et tenta de fusionner l'ISU à la Sailors Union of the Pacific (SUP). Dans l'intervalle, les communistes américains se retiraient de l'ISU pour former un autre syndicat, The National Maritime Union, fondé à New-York en 1936 et affilié plus tard au CIO.

Au Canada, en 1936, les dirigeants du syndicat des marins canadiens ont songé à s'affilier avec la NMU. Ils ont jugé plus prudent, semble-t-il, de collaborer sans affiliation officielle. C'est ainsi que la CSU préféra s'affilier au CMTC et le demeurera jusqu'à son exclusion en 1949. ${ }^{10}$ Ce syndicat fut affilié en outre à l'AFL jusqu'en 1944 bien que très peu de textes de propagande de la CSU en fassent mention. En fait l'AFL n'apporta aucune aide au syndicat des marins canadiens. Une brochure de la CSU explique ainsi le contexte:

It (CSU) started from the ranks without aid from the AFL or any other central trade union body. The AFL seamen's union which had long been in the hands of corrupt leaders, had completely desintegrated. The AFL had no seamen's union while the new CIO was establishing a Seamen's organization ${ }^{11}$.

9 Marge Ferguson, "The Canadian Seamen's Union. An historic chapter in the battle for a union», The Canadian Tribune (February 12, 1975): 5.

10 "Sullivan formed the Canadian Seamen's Union out of the fusion of the Marine Worker's Union of the Great Lakes, based in Toronto, and Montreal's National Seamen's Union (ACCL). According to Sullivan's own explanation, the party needed to work in a larger organization and so the CSU broke off from the ACCL to affiliate with the AFL." A. Lévesque-Olssen, op.cit., 152. A ce sujet, voir aussi H.A. Logan, Trade Unions in Canada: their development and functioning (Toronto, MacMillan, 1948), 288 et Pat Sullivan, Red Sails on the Great Lakes (Toronto, MacMillan, 1955), 14-15.

11 Brochure de la CSU, The C.S.U. and You. The case for free trade unionism (Bibliothèque du Ministère du Travail, Ottawa, s.1., s.d.), 30 pages. 
Jusqu'en 1944, l'affiliation paradoxale de la CSU à l'AFL l'obligea à rester neutre dans la guerre opposant aux États-Unis l'AFL à la National Maritime Union (CIO). La CSU recevra cependant beaucoup d'aide de la NMU. Le journal Searchlight, en retour, après 1944, prend ouvertement parti pour ce syndicat progressiste et contre l'AFL.

Durant les deux premières années d'existence de la CSU, le principal objectif était de gagner l'adhésion de l'ensemble des travailleurs maritimes et d'obtenir la reconnaissance syndicale. Le droit et la nécessité d'un véritable syndicat seront des thèmes repris continuellement par le Searchlight. Dès le printemps 1937, la CSU réussissait à obtenir une entente verbale avec la Canada Steamship Lines, Tree Line et la Quebec and Ontario Transportation, qui accordaient des augmentations de salaires de $40 \%$. La CSU tentera par la suite d'étendre ces gains aux travailleurs des autres compagnies. À l'automne 1937 la menace d'une grève générale permit aux marins des Grands lacs de décrocher des augmentations de 20 à 25\%. Ces victoires accélérèrent ia syndicaiisation, si bien qu'à la nin de 1937, plus de $60 \%$ des marins rejoignaient les rangs de la CSU.

Rapidement l'Union des marins canadiens (CSU) s'implanta autour des Grands lacs; des bureaux furent ouverts non seulement à Montréal et Toronto, mais aussi à Hamilton, Goderich, Collingwood, Midland et Fort William. Avant la fin de l'année 1937, des organisateurs de la CSU étaient en fonction à Terre-Neuve, en Nouvelle-Écosse et au lac Winnipeg. C'est ainsi qu'au deuxième Congrès annuel de la CSU tenu à Montréal en 1938, il y avait plus de 72 délégués et tous les ports des Grands lacs étaient représentés. Pour la première fois, les marins de la base pouvaient discuter librement de leurs problèmes.

La CSU va jouer un rôle important dans la promotion de l'unité des travailleurs organisés. Elle fait souvent appel aux catégories de travailleurs syndiqués reliés aux activités portuaires ${ }^{12}$ (débardeurs, charretiers, manutenteurs de grains, hommes d'entrepôts), pour les aider à organiser les marins non syndiqués, à combattre l'association du capitaine McMaster et à obtenir la reconnaissance syndicale. La CSU, conformément à la ligne politique élaborée à la commission syndicale du PCC, travaille étroitement avec les nouveaux locaux de syndicats internationaux créés vers la même épo-

12 Le Searchlight (1-07-1937): 3; (15-07-1937): 2. 
que, en vue d'établir une vaste "Fédération maritime du transport» qui travaillerait en collaboration avec la National Maritime Union et l'International Longshoremen's Association, comme cela existait en Grande-Bretagne et sur la côte Ouest des États-Unis ${ }^{13}$. Un front commun sera constitué en 1937 avec des locaux de l'AFL, regroupant notamment le syndicat des Marins de la Nouvelle-Écosse, filiale de la CSU.

$\mathrm{Au}$ niveau provincial, la CSU préconise déjà l'établissement d'un secrétariat général permanent des Unions internationales et autres associations ouvrières affiliées au CMTC au Québec. Le rôle de cette fédération qui ne sera créée au Québec qu'en 1952 sera de représenter les affiliés du CMTC auprès du gouvernement provincial.

Pendant toute cette période, la revendication de la journée de 8 heures sera omniprésente. Le Searchlight dénonce, à partir de cas concrets, des situations où des équipages doivent travailler 16 heures par jour, sept jours par semaine, à $\$ 30.00$ par mois ${ }^{14}$. Certains sont obligés de travailler parfois 25 et 30 heures avant de se reposer, sans être payés plus que le salaire ordinaire ${ }^{15}$.

Pour absorber le surplus de main-d'œuvre disponible, la CSU demande aux armateurs de compléter les équipes et d'établir le système des trois quarts. Elle dénonce l'augmentation des accidents causés par un manque de personnel et les trop longues heures de travail. Il n'est pas rare en 1936 de voir des marins travailler 84 heures par semaine et souvent plus de cent heures.

La pratique des armateurs consistant à introduire dans les équipages déjà insuffisants des «jeunes gens mal payés et sans expérience, pour remplacer les hommes d'expérience» est dénoncée, tout comme celle consistant à engager des étudiants ou des amis qui ne travaillent que durant les plus beaux mois de l'été. On dénonce l'embauchage sur les quais et on veut forcer les compagnies à engager les hommes dans les bureaux de l'Union.

Les revendications de mesures de sécurité sont nombreuses: on se plaint particulièrement des explosions aux salles de fournaises, «explosions dues aux gaz, causées par la négligence des propriétaires de vaisseaux à installer des ventilateurs ». ${ }^{16}$

\footnotetext{
13 Le Searchlight (1-08-1937): 2.

14 Brochure du CSU, The C.S.U. and You, 5.

15 Le Searchlight (16-08-1937): 3.

16 Le Searchlight (1-11-1937): 3.
} 
Au chapitre des salaires, le Searchlight compare les conditions des marins canadiens à ceux des États-Unis:

Un homme de pont (deckhand) reçoit ici de \$45. à 50. par mois pour 12 heures d'ouvrage par jour divisé en 2 quarts de 6 heures, et souvent il fait jusqu'à 30 heures sans arrêt, sans rémunération additionnelle. Aux États-Unis, le même manœuvre gagne plus de $\$ 80.00$ par mois pour 8 heures par jour avec un taux de .60 cents l'heure quand il est obligé de travailler plus de huit heures. ${ }^{17}$

\section{II - 1940-1941: la lutte contre la répression gouvernementale}

Pendant la période 1940-41, les affrontements avec les armateurs se multiplient et sont particulièrement violents. La CSU veut étendre à l'ensemble des travailleurs maritimes les augmentations salariales. Cet épisode prendra fin avec le début de l'année 1942 alors que le Parti communiste canadien modifie sa position face à la guerre: Maintenant que les troupes fascistes menacent le premier État ouvrier du monde, il devenait impérieux pour toutes les forces progressistes du monde entier de s'unir pour participer à l'effort de guerre. Alors que de 1939 à 1941, les communistes au Québec opposés à la guerre et à la conscription tentent des rapprochements avec les nationalistes anticonscriptionnistes ${ }^{18}$, après juin 1941 le Parti communiste canadien, bien qu'encore officiellement illégal, se range derrière Mackenzie King pour appuyer l'effort de guerre contre le fascisme. Ce changement de stratégie lui permettra de reprendre ouvertement ses activités.

Mais avant de mettre en ouvre cette tactique de «collaboration»(1942-1945), les dirigeants de la CSU ont subi une répression sauvage de la part de la bourgeoisie canadienne et de son appareil d'État. En décembre 1940 on comptait, selon le Searchlight «une vingtaine de trade-unionistes placés arbitrairement dans des camps de concentration sans procès, en vertu des 'Règlements de Défense du Canada'.${ }^{19}$ En avril 1940 , leur nombre atteignait 70 . Le président de la CSU, Pat Sullivan et le secrétaire Jack Chapman furent emprisonnés quelques mois. Dave Sinclair, éditeur du journal Searchlight

17 Le Searchlight (16-08-1937): 3.

18 À ce sujet, voir Marcel Fournier, Histoire et idéologie du groupe canadien-français du parti communiste (1925-1945). Thèse de M.A. (U. de M., 1969), quatrième partie: 222-285.

19 Le Searchlight (1-01-1941): 7. 
et Charles Murray, directeur de l'organisation dans la province de Nouvelle-Écosse, furent également détenus sans accusation. Au début de janvier 1941, la lutte pour la libération des chefs syndicaux s'accentua; des conférences de protestations seront organisées à travers le pays en mars et en avril. Sullivan était interné à cause de sa participation active à la grève du printemps 1940. Accusé d'appartenir à une "organisation déloyale», Sullivan signa un affidavit affirmant qu'il n'avait jamais été communiste. Après 15 mois d'emprisonnement, on l'accusait d'avoir entretenu des rapports avec le Parti communiste canadien ${ }^{20}$.

Réélus à leur poste respectif, ils recevront l'appui de centaines de comités de défense «Pat and Jack» qui populariseront la cause de ces officiers syndicaux chez les 5,000 syndiqués de la CSU et même chez tous les syndicats affiliés au Congrès des métiers et du travail du Canada.

$\mathrm{Au}$ début de la guerre, les effectifs de la marine baissèrent dangereusement, les marins préférant s'engager dans les industries de guerre où les salaires étaient plus intéressants. Beaucoup délaisseront la marine marchande pour s'intégrer aux forces armées. L'hémorragie est telle qu'au printemps 1941, la CSU fait pression pour que le "gouvernement libère les marins du service actif et les renvoie au service sur les Grands lacs» ${ }^{21}$. La CSU exige une loi pour interdire les équipages réduits. Avec cette baisse des effectifs, la revendication de la journée de 8 heures et du système des trois quarts qui étaient parmi les principales demandes avant 1939, sera temporairement placée au second rang durant la guerre. La lutte pour la reconnaissance effective du syndicat se poursuit. Durant toute cette période, on dénonce la pratique patronale consistant à engager des marins en dehors du local de l'Union. Les marins tenaient au système rotatif très efficace mis au point par le syndicat.

Avec la guerre, plusieurs revendications concernent l'augmentation des mesures de sécurité sur les navires. Par exemple: la construction de portes ouvrant sur les ponts pour toutes les cabines, des équipements de sauvetage en nombre suffisant, l'obligation pour les officiers de posséder un certificat de compétence, une application plus stricte des exercices avec chaloupes de sauvetage, l'installation de palans uniformisés, l'installation des «sans-fil» à bord de tous les

20 Le Searchlight (novembre 1941): 1.

21 Le Searchlight (avril 1941). 
vaisseaux sans égard à la grosseur du tonnage. Le syndicat souhaite que tous les bateaux canadiens disposent de plus de personnel diplômé. Pour améliorer les conditions de vie des équipages, la CSU revendique diverses mesures sanitaires comme la fumigation annuelle des cabines ou l'inspection bi-annuelle des cuisines et celle des glacières à tous les 3 mois.

Au 4 e congrès annuel de la CSU, en 1941, on retrouvera parmi les résolutions la demande de participation à l'assurance-chômage et les facilités pour les marins d'exercer le droit de vote.

La solidarité et le militantisme qui caractérisaient la CSU, particulièrement durant ces années, ont forcé les employeurs à accorder d'importantes augmentations salariales. Le salaire moyen des marins a augmenté de presque $85 \%$ entre 1936 et 1940 . Ainsi un matelot qui gagnait \$36. par mois avant l'existence de la CSU obtenait une fois syndiqué, en moyenne, $\$ 40$. au printemps 1937 , $\$ 50$ à l'automne 1937 , $\$ 55$. en 1938 et $\$ 62.50$ en 1940 . Un cuisinier qui obtenait de \$85. à \$95. mensuellement en 1936 gagnait en moyenne entre \$117.50 et $\$ 127.50$ en 1940.

Évidemment, en 1939, plusieurs compagnies essayèrent d'utiliser le prétexte de la guerre pour retarder la signature des nouveaux contrats. En avril 1940, plusieurs compagnies des lacs refusaient encore de reconnaître l'Union des marins ${ }^{22}$.

Le Bureau de conciliation du Ministère du Travail accordera, en avril 1940, d'importants avantages aux 5,000 marins des Grands lacs: en premier lieu la reconnaissance syndicale. Le gouvernement demandera aux diverses compagnies de reconnaître la CSU comme «seul agent de discussion collective». Au chapitre salarial, une augmentation de $\$ 10$. par mois pour les huileurs et de $\$ 7.50$ pour toutes les autres catégories est accordée avec rétroactivité à l'ouverture de la saison de 1940. Le travail du dimanche est supprimé et les compagnies doivent payer les coûts de transport des travailleurs, à la fin de la saison, entre le port d'attache et le lieu de résidence du marin.

22 Parmi celles-ci retenons: les navires «Misener» (23 navires), Gulf and Lake Navigation (3), Beaubré Line (49), Lakehead Transportation Co. (9) et Paterson Steamship Co. (30). Les compagnies d'alors qui reconnaissaient l'Union étaient: Canada Steamship Lines (73 navires), Upper Lakes \& St-Lawrence (35), Quebec and Ontario Navigation (7), Algoma Central Steamships (4), North West Steamships (2) et Union Transit (1). 
C'est à partir de 1940 en effet que figurent parmi les avantages économiques les «bonis de vie chère» et les «bonis de guerre». Ces derniers ne furent attribués au départ qu'aux seuls équipages à destination des ports antillais. En 1942, on revendiquera la généralisation de ces bonis.

\section{III - 1942-1945: effort de guerre et collaboration tactique}

À partir de 1942, et jusqu'à la fin de la guerre, la CSU se déclare prête à collaborer entièrement avec le gouvernement dans son effort de guerre. Cette collaboration tactique de la CSU avec le gouvernement et les armateurs en vue d'assurer «l'harmonie sur le front de la production" apparaissait essentielle aux yeux des militants syndicaux communistes pour qui l'objectif premier était la destruction du fascisme.

Dans son Programme de la Victoire pour la navigation intérieure et maritime, présenté au gouvernement en 1942 et dans son Mémoire au Gouvernement du Canada, présenté en mai 1944, la CSU propose au gouvernement central diverses réformes afln d'améliorer les conditions de travail, stabiliser la production et accélérer les échanges commerciaux. La CSU formule une politique de transport pour le Canada consistant à augmenter les effectifs de la marine marchande canadienne après la guerre «afin de garantir qu'elle jouera le rôle qui lui revient de droit dans le commerce mondial» ${ }^{23}$.

Cette tactique de collaboration permit à la CSU de poursuivre son travail de syndicalisation et d'obtenir certains gains au chapitre salarial. Cette tactique est cependant aujourd'hui vigoureusement contestée par des militants marxistes-léninistes qui œuvrent à édifier un parti révolutionnaire de type nouveau tout en revendiquant l'héritage de ces communistes canadiens:

Le Parti Communiste lutte pour que les syndicats concluent avec des patrons des accords anti-grève. Ces clauses enlevaient aux travailleurs le droit de faire la grève en échange de quelques compensations au chapitre de la sécurité d'emploi et autres peccadilles (sic). Aux yeux des militants communistes, cette politique favorisait l'effort de guerre national. Pourtant, la lutte des classes dans le pays n'était pas éteinte pour autant. Cette tacti-

23 Mémoire présenté par la Canadian Seamen's Union au Gouvernement du Canada, 30 mai 1944 (Ministère du Travail, Ottawa), 16. 
que mécanique et incorrecte ( $\mathrm{sic}$ ) contribua encore plus à isoler le parti des masses qui constataient avec raison que pendant qu'elles travaillaient à mort pour l'effort de guerre, les riches s'enrichissaient comme jamais et se voyaient accorder des privilèges inouïs par le gouvernement. ${ }^{24}$

Avant de s'en prendre à l'analyse de la situation internationale faite par Dimitrov et à sa juste stratégie de «front populaire» pour combattre le fascisme, et avant d'accuser rapidement le Parti communiste d'appliquer mécaniquement les directives de la $3^{\mathrm{e}}$ Internationale, ne faudrait-il pas étudier de plus près ce type d'appui au gouvernement bourgeois dans son contexte historique?

Le journal Searchlight fait largement état, durant ces années, des développements quantitatifs de l'Union des marins. En 1941, la CSU se fusionne avec la Canadian Fishermen's and Fishlander's Union (CFFU) de la Nouvelle-Écosse. Le Searchlight devient alors l'organe officiel de la CSU et de la CFFU ${ }^{25}$. Dès juin 1942, l'Union lançait une campagne de propagande en vue d'organiser les équipages des navires-citernes. En avril 1943, on fait état de la réussite de la campagne de syndicalisation destinée à tous les marins de hautemer. La même année, la CSU organisait les marins de la côte ouest. Enfin, en septembre 1944, la Deep Sea and Inlandboatmen's Union of the Pacific Coast décidait de se fusionner avec l'Union des marins canadiens.

On se rappelle que la CSU s'était affiliée à l'International Seamen's Union (ISU). Ce dernier syndicat n'était pas implanté au Canada. En septembre 1944, la CSU rompt cette affiliation suite au refus de l'ISU de modifier les conditions de son affiliation. D'après le Searchlight, l'ISU imposait à la CSU la chasse aux communistes. On voulait obliger la CSU à prendre position contre la NMU, à s'engager à combattre tous les «communistes» à l'intérieur du syndicat CSU, et à combattre tous «les partis communistes du continent». Or, comme la NMU n'avait jamais attaqué la CSU et avait même collaboré étroitement avec elle, la CSU résilia sa charte, d'autant plus que l'AFL, comme le répéta maintes fois le Searchlight, n'a jamais fourni quelque aide que ce soit aux marins de la CSU ${ }^{26}$.

L'année 1944 marque donc le début de l'offensive de l'AFL contre l'Union des marins canadiens (CSU). L'AFL décide alors de

24 Mobilisation (revue), 4, no 4: 17.

25 Le Searchlight (15-08-1941): 1.

26 Le Searchlight (septembre 1944). 
lancer un autre syndicat au Canada, la Seafarers International Union of North America (SIU) qui travaillera après la guerre à la destruction de la CSU.

De même au Congrès du CMTC de 1946, tenu à Hamilton, l'exécutif demandera à la Fédération américaine du Travail de faire cesser les agissements des dirigeants canadiens de la SIU. La CSU a réussi assez bien pendant la guerre à maintenir le nouveau venu dans l'inactivité, jusqu'à l'arrivée de Hal Banks au Canada ${ }^{27}$.

En 1939 les navires d'outre-mer naviguant sous le drapeau canadien étaient peu nombreux: entre 8 et 13 seulement. Le gouvernement canadien se mit alors à construire des navires marchands afin d'acheminer rapidement les marchandises. La CSU syndicalisera plusieurs de ces groupes de marins qui naviguaient sur les parcours les plus risqués. À la fin de 1942 la compagnie de la couronne Park Steamship Company mettait en opération entre 283 et 300 bateaux ${ }^{28}$. À partir de 1943 ces navires furent remis à l'entreprise privée et entre 1943 et 1949 furent revendus à des compagnies subsidiaires étrangères. Les armateurs pouvaient obtenir plus facilement de la main-d'œuvre à bon marché pour les bateaux non-canadiens. De plus, la législation canadienne concernant les mesures de sécurité, ne s'appliquant que pour les navires canadiens, les patrons avaient intérêt à liquider la marine canadienne.

C'est ainsi qu'à partir de 1943 , de nombreux témoignages de marins et discours officiels de délégués syndicaux mettent en lumière la nécessité pour le Canada de disposer d'une marine marchande permanente. Non seulement le Canada devrait-il maintenir les 200 navires en service en 1945, mais il devrait, disent-ils, en faire construire 200 autres. Plus la guerre semble sur le point de s'achever, plus le Searchlight rappelle «le courage, la loyauté et l'utilité des équipages de la marine marchande canadienne durant les hostilités » ${ }^{29}$. Le Mémoire de 1944 adressé au gouvernement est très explicite. La CSU s'inquiète de ce qui adviendra du transport maritime après la guerre et du chômage qu'entraînera cette liquidation de la marine canadienne:

27 Charles Lipton, The Trade Union Movement (Montreal, Canadian Social Publication, 1966), 276.

${ }_{28}$ À ce sujet, voir Marge Ferguson, "The C.S.U. An historic chapter in the battle for a union», The Canadian Tribune (February 12, 1975), 5. 1945.

29 Voir en particulier les numéros du Searchlight de juin 1943, avril et mai 
Il nous faut (la marine marchande canadienne) pour maintenir notre industrie maritime, pour fournir de l'ouvrage à des mililiers de marins (...) et pour encourager le développement des échanges commerciaux entre le Canada et d'autres pays (...) Nous proposons donc au gouvernement d'ajuster sans retard son programme de construction de navires rapides et bien construits. ${ }^{30}$

La CSU proposera, dans le même sens, l'établissement par le gouvernement d'une école de formation d'officiers canadiens. La majorité des officiers provenaient de la marine marchande britannique, ce qui causait, aux dires des marins, «des malentendus inévitables entre ces officiers et les marins ».

Ce Mémoire de 1944 résume les principales améliorations que la CSU espère pouvoir obtenir du gouvernement dans un esprit de collaboration en reconnaissance du fait que «les relations entre nous et le Ministère des Transports et le Ministère des Munitions et du matériel et la mesure de collaboration entre armateurs et marins qu'on a réussi à atteindre, ont joué un très grand rôle dans le renforcement de l'industrice" 31 .

La CSU réclame d'abord que toutes les compagnies soient tenues d'engager leur personnel par l'entremise des dépôts de personnel. Cette demande sera toujours l'objet d'une négociation avec les compagnies, le gouvernement refusant d'intervenir malgré la démonstration du syndicat à l'effet que cette pratique permettrait de stabiliser la situation du personnel de la marine.

On veut, dans tous les ports de mer où se trouve un dépôt de personnel, l'établissement d'un «comité d'appel» où serait représenté la CSU et un représentant du gouvernement qui pourrait entendre les griefs et en disposer rapidement. Ici encore, le gouvernement ne pouvait s'engager de la sorte.

La CSU demande une représentation de marins sur les divers conseils qui touchent l'industrie de la construction navale afin de faire connaître le point de vue syndical. Pour remédier aux fluctuations dans le personnel de l'industrie maritime, le mémoire recommande au gouvernement d'établir des examens de qualification pour toutes les catégories de travailleurs. Le Searchlight accorde une grande importance aux plaintes concernant la mauvaise qualité des repas. «Plus de $50 \%$ des griefs des hommes proviennent d'une mau-

30 CSU, Mémoire présenté au gouvernement fédéral, en 1944, 14-15.

31 CSU, Mémoire de 1944, 4. 
vaise cuisine et d'aliments mal préparés,» peut-on lire dans le $M e ́$ moire de 1944.

Au chapitre des salaires, la «collaboration» de la CSU avec le gouvernement lui permit d'obtenir certains gains. Le Mémoire de 1944 reconnaît que «le gouvernement a adopté quelques-unes des mesures que nous avions préconisées pour améliorer la situation des hommes: l'augmentation des salaires de base, l'allocation d'une prime de risque de guerre et l'exemption d'impôt sur le revenu, des allocations de pension et des primes de risque de guerre» ${ }^{32}$. Par contre, devant les multiples demandes de la CSU voulant que les "Unions de compagnies soient déclarées hors la loi afin de faire disparaître l'une des principales causes de confusion et de désaccord dans les relations industrielles ${ }^{33}$, le gouvernement resta muet.

L'inflation touchant tous les travailleurs, la CSU exige alors que les bonis de vie chère soient payés à tous les ouvriers. Quant aux bonis de guerre, la CSU demande qu'ils soient uniformisés à $\$ 2.00$ par jour pour tous. Les moins de 21 ans recevaient $\$ 22.25$ par mois et les autres $\$ 44.50$. La CSU défend le principe «à risque égal, compensation égale».

Dès avril 1944, l'Union des marins demande aux armateurs de s'engager immédiatement à accorder 6 mois après la fin des hostilités la journée de huit heures et à relever les salaires de base de $\$ 10.00$ par mois pour toutes les catégories avec la possibilité d'exécuter en temps supplémentaire tout «travail non-nécessaire». Ils revendiquent le temps supplémentaire pour le travail exécuté les jours de fête. Le Searchlight mène une campagne pour que les salaires des travailleurs maritimes soient le plus près possible des salaires offerts dans les industries de guerre où se trouvent les mêmes métiers. Même avec l'augmentation de $\$ 10.00$ demandée pour l'après-guerre les salaires ne dépasseront pas 40 cents l'heure pour les ouvriers qualifiés sur mer. Les ouvriers qui ont un métier identique à terre recevaient déjà en mai 1944 un salaire qui variait de .60 cents à .80 cents l'heure.

Les marins étaient très insatisfaits du «consortium des équipages» établi par le gouvernement afin de centraliser les ressources disponibles. Les marins ne s'enrôlent pas en grand nombre dans ce système parce qu'en signant, ils abandonnent le droit de choisir leur na-

CSU, Mémoire de 1944: 4.

33 Le Searchlight (mai 1944): 3. 
vire, n'ont pas de représentant syndical à ce «pool» et ne peuvent par conséquent formuler de grief concernant leur sécurité d'emploi. Ce système les désavantageait également au plan salarial. Dans ce consortium, les «salaires de base» étaient également ceux qui prévalaient sur les navires anglais. Or ceux-ci étaient très inférieurs à ceux offerts en Amérique du Nord, en Norvège, à Panama et dans quelques autres marines (tableau I).

\section{TABLEAU I}

Salaires mensuels de base en 1942

\begin{tabular}{lccrr}
\multicolumn{1}{c}{$\begin{array}{c}\text { Canada } \\
\text { Catégorie }\end{array}$} & $\begin{array}{c}\text { Marine } \\
\text { Lacs }\end{array}$ & $\begin{array}{c}\text { marchande } \\
\text { de G. }- \text { B }\end{array}$ & $\begin{array}{c}\text { M. de } \\
\text { Panama }\end{array}$ & \multicolumn{1}{c}{ M. des } \\
Marin qualifié & $\$ 100.00$ & $\$ 47.07$ & $\$ 90.00$ & $\$ 100.00$ \\
Marin ordinaire & 75.50 & 32.67 & 72.50 & 82.50 \\
Charnentier & & 78.63 & 102.50 & 112.50 \\
Chargeur d'eau & 100.00 & 51.50 & & 110.00 \\
Chaufferie & 93.00 & 51.50 & 92.50 & 102.50 \\
Magasinier & & 53.71 & & 110.00 \\
Chef-steward & 135.00 & 106.87 & 137.50 & 147.50 \\
Cuisinier & & 89.15 & 122.50 & 132.50
\end{tabular}

L'indemnité de guerre était généralement plus haute sur les navires américains, canadiens, norvégiens et panaméens. Les salaires de base de la marine anglaise sont aussi plus bas que ceux en vigueur sur les Grands lacs et ces derniers étaient du reste nettement

TABLEAU II

Salaires mensuels obtenus en 1943

\begin{tabular}{lccc}
\multicolumn{1}{c}{ Catégorie } & Avant 1943 & Avant 1943 & En 1943 \\
Charpentier & $10,000 \mathrm{~T}$ & $4,700 \mathrm{~T}$. & \\
Bosun & $\$ 70.00$ & $\$ 68.25$ & $\$ 70.10$ \\
Marin qualifié & 64.50 & 59.85 & 64.50 \\
Donkeyman & 56.20 & 52.20 & 56.20 \\
Chauffeur & 62.90 & 59.15 & 62.90 \\
Second cuisinier & 58.50 & 54.70 & 58.50 \\
Asst-steward & 49.50 & 43.50 & 49.50 \\
\hline
\end{tabular}


inférieurs à ceux offerts sur les navires américains pour le même service.

Parmi les principales victoires syndicales obtenues pendant ces trois dernières années de guerre, rappelons celle de juillet 1943 par laquelle la CSU faisait accepter, par la Compagnie Park et la $\mathrm{Ca}$ nada Steamship, pour la première fois, le salaire égal sans égard au tonnage (tableau II).

En 1943, le travail supplémentaire était payé au taux de $\$ 0.50$ l'heure et la pension aux marins était de $\$ 30.00$ par mois et dégrevée d'impôt.

Enfin, le 3 mai 1945 un contrat signé entre les Pétroliers Park et la CSU apportait des augmentations salariales de \$5. à \$10. par mois pour toutes les catégories. Le temps en surplus était payé 75 cents de l'heure. C'était la première fois que les pétroliers étaient sous contrat avec la $\mathrm{CSU}^{34}$.

\section{IV. - 1945-1946: la grève de 1946 et les débuts de l'offensive anti- ouvrière}

Dans l'immédiat après-guerre, des grèves décisives furent menées au Canada, notamment chez Ford à Windsor (obtention de la formule Rand), chez Stelco à Hamilton et chez les marins des Grands lacs à l'été 1946. Dans toutes ces grèves, les syndiqués communistes ont joué un rôle important. Ces grandes victoires du mouvement ouvrier ont été immédiatement suivies d'une vaste offensive antiouvrière sous le couvert d'une vigoureuse campagne anticommuniste. L'emprisonnement par les gouvernements, notamment celui de $\mathrm{Du}$ plessis, des dirigeants de la CSU et des leaders du syndicat des Textiles s'inscrit dans ce contexte des débuts de la «guerre froide».

Avant d'étudier cette grève de 1946, voyons les principales revendications de la CSU durant cette période. Dans son programme d'après-guerre, les trois points sur lesquels le Searchlight insistera davantage sont: le maintien d'une marine marchande, le maintien des salaires au niveau de 1945, et la journée de 8 heures pour les marins des côtes et des lacs ${ }^{35}$.

\footnotetext{
34 Voir l'échelle complète des salaires dans le Searchlight (5 juin 1945): 1.

35 Le Searchlight (8 septembre 1945): 1.
} 
La campagne intense pour le maintien de la marine prit diverses formes: pétitions, assemblées de masse, rencontres avec des députés, cartes postales au premier ministre, délégations de marins au Parlement, mémoires au gouvernement ${ }^{36}$. Face à la menace du chômage, on fait valoir tous les avantages d'une marine proprement canadienne. On demande encore l'ouverture d'une école pour former des «hauts gradés canadiens», les officiers pendant la guerre étant très majoritairement britanniques. On réclame des «gratifications pour service de guerre» aux marins marchands au même titre que ceux de la marine royale pour le temps passé sur le théâtre des opérations militaires.

D'une façon générale l'Union cherche à incorporer dans les conventions avec les armateurs tous les avantages obtenus dans les autres secteurs industriels à l'époque: assurance-maladie et vacances payées en particulier. Dans sa lutte pour la journée de huit heures, la CSU compare toujours la situation des marins à celle des autres travailleurs industriels.

Pour améliorer leurs conditions de travail, les marins souhaitent la généralisation de l'utilisation de l'huile (engins Diesel) soulignant les désavantages du charbon. On accepte de moins en moins de travailler dans les chambres de chauffage à des températures de 150 à $165^{\circ} \mathrm{F}$. On peut lire dans le Searchlight: "Vous trouverez le plus souvent chaque chauffeur et soutier à l'hôpital au cours des tournées dans les tropiques.» ${ }^{37}$ On blâme l'indifférence des capitaines et l'impossibilité d'obtenir des soins dans certains ports. On rapporte un nombre élevé de morts suite à ces difficultés ou négligences ${ }^{38}$.

Au chapitre salarial, dès le début de 1946, la CSU revendique un salaire minimum de 50 cents de l'heure pour les marins ordinaires. Cette revendication prenait en considération les salaires payés aux ouvriers canadiens ne travaillant pas sur mer:

Comment expliquer qu'un chauffeur à bord d'un bateau ne reçoit que $\$ 136.93$ par mois alors que sur terre, il recevrait $\$ 50.48$ par semaine de 48 heures? Comment expliquer que les boulangers sur un navire ne touchent que $\$ 149.00$ par mois, ou $\$ 0.62$ de l'heure, alors que sur terre, ils toucheraient en $1944 \$ 0.68 / \mathrm{h}$. ? Un peintre sur un navire gagne $\$ 0.471 / 2$ à $\$ 0.61 / \mathrm{h}$ alors que sur

36 Le Searchlight (octobre 1945): 1.

37 Le Searchlight (18 juin 1945): 4.

38 Le Searchlight (15 août 1945). 
terre, il gagnerait $\$ 0.96 / \mathrm{h}$. Un charpentier a sur la mer $\$ 0,64^{1 / 2} \mathrm{~h}$. alors que sur terre, il obtiendrait $\$ 1.12$ l'heure ${ }^{39}$.

En 1945, un simple marin (haute-mer) gagnait \$114. par mois: la CSU revendiquait pour lui $\$ 120$. Pour le marin qualifié qui recevait $\$ 134$. en 1945 , la CSU revendiquait $\$ 140$. Parmi les mieux rémunérés: un chef cuisinier qui gagnait \$166: par mois, la CSU demandait $\$ 171$. Au $6^{\mathrm{e}}$ Congrès de la CSU tenu en mars 1946, on formula la même demande pour les marins des Grands lacs.

La grève de 1946 a été particulièrement bien couverte par le Searchlight. Un supplément illustré de ce journal nous permet de constater la brutalité des affrontements.

À ce $6^{\mathrm{e}}$ Congrès, la CSU exprimait ses deux grands objectifs : la journée de 8 heures et le salaire minimum de 50 cents l'heure. Une grande campagne pour populariser ces demandes fut poursuivie en mars et avril: «Enterrons la semaine de 84 heures», «Non à la journée de 12 heures!».

Le 16 mai, le Searchlight annonce les résultats d'un vote d'avis de grève pour obtenir la journée de 8 heures sur mer et sur les lacs. Cet objectif sera réduit par la suite à 48 heures sur mer et 40 heures amarré. Le 25 mai la grève prenait effet pour tous les navires.

Dès le début les syndiqués durent affronter les policiers qui protégeaient les briseurs de grève en leur permettant de monter sur les navires. Au début de juillet, 130 marins de la CSU et 5 officiers avaient été emprisonnés depuis le début de la grève. À la fin du mois d'août, le nombre de marins emprisonnés était passé à 230 . Ces arrestations avaient été effectuées lorsque les syndiqués tentaient de monter «illégalement» à bord des navires pour y déloger les briseurs de grève ${ }^{40}$. Après 28 jours de grève, certaines compagnies acceptaient d'accorder la journée de huit heures. Mais quelques semaines plus tard les trois plus importantes compagnies des Grands lacs refusaient de respecter leur contrat. Le Searchlight exige qu'Ottawa surveille les armateurs et fasse respecter les contrats ${ }^{41}$. Le Searchlight fait largement écho aux incidents de Cornwall qui se sont produits pendant la grève et qui constituaient une véritable provocation à l'endroit de la CSU; un navire était alors immobilisé dans le canal. Dans la nuit du 31 mai, un groupe de 75 «assommeurs profes-

39 Le Searchlight (janvier 1946).

40 Le Searchlight (23 août 1946): 1.

41 Le Searchlight (1er août 1946): 1. 
sionnels» (briseurs de grève) du capitaine McMasters, financé par la Canada Steamship Lines, pillèrent restaurants et magasins de Cornwall pour que le blâme en soit jeté sur les marins. Il s'ensuivit des émeutes aux cours desquelles 300 policiers de la Royal Canadian Mounted Police (RCMP) durent quitter les lieux sous la pression des citoyens qui avaient endossé les revendications des syndiqués. L'organisation de McMasters qui continua de fournir des «scabs» aux armateurs fut constamment la cible du journal durant tout l'été. Finalement, le 3 septembre, les trois compagnies acceptaient d'accorder des augmentations considérables, allant jusqu'à $20 \%{ }^{42}$.

La CSU avait reçu durant toute cette période l'appui d'un nombre considérable de syndicats locaux ${ }^{43}$, du Congrès des métiers et du travail du Canada et de la National Maritime Union ${ }^{44}$.

Parallèlement à cette grève, une vaste campagne pour défendre les marins en procès et obtenir leur libération se poursuivra durant l'été et l'automne. Parmi les accusés, Harry Davis, présidentintérimaire de la CSU, Cyril Lenton, trésorier, et Conrad Sauras, directeur de l'organisation de la CSU à l'échelle nationale furent accusés d'avoir transgressé le "Canada Shipping Act», loi caduque qui accordait des peines d'emprisonnement à tous ceux qui s'introduisaient sur les bateaux sans la permission du capitaine. A Montréal, il y aura le procès de Dan Daniels, rédacteur du Searchlight, de J.-P. Paradis et de Robert Sénécal ${ }^{45}$.

Le 28 novembre 1946 Cyril Lenton sera condamné à 3 mois d'emprisonnement et Conrad Sauras à 6 mois. Parallèlement Duplessis obtenait la condamnation à 6 mois d'emprisonnement de Kent Rowley, directeur de l'organisation des Travailleurs unis du textile d'Amérique, pour sa participation à la grève de 100 jours à Valleyfield, à l'automne 1946.

En décembre plusieurs furent acquittés dont Davis, Tessier et Grabek $^{46}$. John Thompson, un simple marin, sera condamné à 3 ans d'emprisonnement ${ }^{47}$. Tous ces procès sont alors situés par le Searchlight dans le mouvement général de répression antiouvrière. Le

42 Le Searchlight (5 septembre 1946).

43 Le Searchlight (10 août 1946): 2.

44 Voir la liste de tous les syndicats dans le Searchlight (23 août 1946).

45 Le Searchlight (14 novembre 1946): 1.

46 Le Searchlight (12 décembre 1946): 1.

47 Le Searchlight (26 décembre 1946): 1. 
journal met de plus en plus les marins en garde contre l'anticommunisme, instrument utilisé par la bourgeoisie canadienne pour diviser la classe ouvrière et justifier la répression contre cette dernière. Devant les tentatives de la bourgeoisie canadienne pour isoler les chefs syndicaux de la CSU et couper l'avant-garde de l'ensemble des marins, le journal Searchlight fait la preuve de la solidarité des syndiqués. Les témoignages d'appuis, les lettres et pétitions et même des poèmes révolutionnaires en faveur des chefs emprisonnés et contre le fascisme se multiplient. Le journal de la CSU informe davantage sur l'ensemble du mouvement ouvrier canadien et américain.

L'objectif principal qui a mobilisé les syndiqués jusqu'à la fin de l'automne fut la modernisation de «l'Acte maritime du Canada» et particulièrement l'abolition de l'article 239 de cette loi qui permettait d'imposer des peines d'emprisonnement aux marins qui s'introduisaient sans permission sur les navires. La lutte contre l'emprisonnement des militants syndicaux et pour la libération des dirigeants du syndicat est liée à la dénonciation de cette arme puissante dont disposaient les armateurs.

Après la longue grève des Grands lacs qui prit fin le 3 septembre 1946, les trois compagnies qui avaient manifesté le plus d'obstination (la Canada Steamship Lines, Paterson et Misener) se virent forcées d'accorder des contrats que la CSU qualifia de la plus grande victoire syndicale maritime. Ce contrat accordait 20\% d'augmentation sur les salaires, une vraie journée de huit heures avec système de 3 équipes, le temps supplémentaire à 75 cents l'heure pour tous les grades, la sécurité syndicale, dix jours de vacances payés par saison et le droit d'ancienneté et de promotion.

Pendant la grève des marins des Grands lacs, des contrats très avantageux étaient signés pour les équipages des pétroliers de la compagnie Park. Des augmentations salariales allant jusqu'à $\$ 10.00$ par mois et rétroactives à août 1945 étaient obtenues. La grève des Grands lacs s'étendra à l'Atlantique. Par la suite, une entente nationale sera signée pour les océaniques. Cette entente qui sera obtenue peu après celle des Grands lacs, entraînera des augmentations d'environ \$15.00 par mois auxquelles s'ajouteront des bonis de guerre de $\$ 44.50$ par mois.

En novembre 1946, un sommet était atteint au plan salarial lorsque la CSU réussit à obtenir de la compagnie des pétroliers des Grands lacs, la compagnie Shell Canadian Tankers, une échelle de 
salaires qui accordait $\$ 118.00$ mensuellement au simple matelot, $\$ 150.00$ au responsable des fournaises et $\$ 195.00$ au cuisinier.

Toutes ces conquêtes acquises grâce à la solidarité et le militantisme des marins de la CSU amènent la bourgeoisie à réagir. Pour elle, tous les moyens seront bons pour briser l'organisation syndicale, y compris la corruption de leaders syndicaux.

\section{V-1947-1949: l'offensive anticommuniste}

Après la guerre, la lutte anticommuniste s'étendit partout en Amérique du Nord ${ }^{48}$. Il fallait intégrer les syndicats à l'appareil d'État afin d'éliminer tout danger de contestation interne au moment où l'impérialisme américain prenait son essor partout dans le monde: en Europe avec le plan Marshall, en Amérique du Sud, en Asie, en soutenant les Japonais et Tchang Kaï-Chek contre les communistes chinois. La répression ne sera pas limitée au milieu syndical. La bourgeoisie alimente toute une campagne de conditionnement idéologique pour déraciner toute idée progressiste chez les masses. «L'Église catholique, le système d'éducation, les organismes de sports et de loisirs, les films, la radio, les publications de toutes sortes, contribuent à cet effort concerté pour provoquer une hystérie collective anticommuniste. ${ }^{49}$

Pour discréditer les partis communistes américain et canadien, la classe dirigeante utilise les accusations d'espionnage: aux ÉtatsUnis les époux Rosenberg sont envoyés à la chaise électrique alors qu'au Québec, le député fédéral Fred Rose est condamné pour espionnage et déporté après sa peine.

La bourgeoisie pourra compter sur de nombreux chefs syndicaux. On écrira :

De plus, la bourgeoisie réussit à corrompre de nombreux chefs

syndicaux quand elle n'en installa pas carrément à la place des

48 Charles Halary a publié, dans les «Cahiers de recherches marxistes révolutionnaires", Le mouvement ouvrier québécois; l'intégration syndicale par l'anticommunisme 1946-1954, 49 pages. L'auteur, un militant trostkyste, décrit la lutte anticommuniste, affirmant qu'elle était une condition sine qua non de l'intégration harmonieuse des syndicats à l'appareil d'État. Il ne montre pas comment l'impérialisme américain, en train de s'implanter en Europe (plan Marshall), en Amérique du Sud, en Asie, avait besoin de consolider ses arrières solidement en éliminant tout danger de contestation interne. Voir à ce sujet, Mobilisation, 4 no 4, "Perspectives historiques sur le Parti communiste du Canada», en particulier le chapitre 4: 19 à 23.

49 Mobilisation, 4 no 4: 20. 
authentiques leaders ouvriers, afin de mieux contrôler le mouvement syndical et de frapper les militants sincères qui défendaient les intérêts de la classe ouvrière.

Aux États-Unis, les John Lewis et Tony Boyle (mineurs), Léonard Woodcock (TUA), Jimmy Hoffa (Teamsters), I.W. Abel (Métallos) et bien sûr Georges Meany (actuel président de l'AFL-CIO) et son adjoint Jay Lovestone (directeur des relations internationales de l'AFL-CIO) et ancien dirigeant communiste maintenant vendu à l'impérialisme US ont entrepris dans les années 40 et 50 une lutte à outrance contre les dirigeants communistes dans les syndicats, aidés par la CIA, le FBI et les capitalistes.

La purge achevée, Meany et Cie ont tout simplement vendu l'AFL-CIO à l'impérialisme américain en supportant la guerre au Vietnam, par exemple, et en organisant la corruption du mouvement syndical partout dans le monde. Ils ont même collaboré avec l'État bourgeois lorsque celui-ci a passé les lois les plus réactionnaires de l'histoire américaine contre les syndicats: la loi Taft-Hartley (1947) qui permet la mise en tutelle des syndicats par l'État, impose un préavis de 60 jours avant toute grève, abolit l'atelier fermé, permet au patron de contester le droit du syndicat de représenter les ouvriers même une fois accrédité et permet de congédier tout militant soupçonné d'être communiste. ${ }^{50}$

Dans sa campagne pour discréditer les leaders ouvriers supposément «déloyaux», la bourgeoisie a réussi à gagner l'appui des dirigeants ouvriers réactionnaires. La CSU est une cible privilégiée dans cette campagne de calomnies anticommunistes. C'est l'époque où la presse bourgeoise multiplie les charges contre les militants progressistes. Un seul exemple rapporté par le Searchlight: le Financial Post du 2 août 1947 demande au gouvernement de «faire le nettoyage» dans les 14 unions suivantes:

Les Ouvriers Unis de l'Électricité (CCT-COI)

Les Ouvriers Unis de l'Automobile (CCT-COI)

Les Ouvriers Unis de la Fourrure et du Cuir (CCT-COI)

Les Ouvriers Unis des Mines et Fonderies (CCT-COI)

La Fédération de la Colombie Canadienne des Ouvriers des Chantiers Maritimes (CCT)

50 L'Anti-communisme, cahier no 15 , supplément au no 45 (vol. 3 no 4), (25 septembre 1975) du journal En lutte. On lira l'ouvrage de Filip Kota, Deux lignes opposées dans le mouvement syndical mondial, pour de plus amples détails sur la corruption des syndicats américains, européens et africains par l'AFL-CIO et la CIA. 
Les Ouvriers Unis du Bois d'Amérique (CCT-COI)

Les Ouvriers Unis des Bureaux (COI)

L'Union des Marins Canadiens (CSU)

Les Ouvriers Unis du Textile d'Amérique (CMT-FAT)

Les Ouvriers des Chantiers et des Scieries

L'Union des Pêcheurs Canadiens (CMT)

Les Pêcheurs Unis et Unions Affiliées (CMT-FAT)

Dans ce contexte, l'Union des marins canadiens devra lutter contre les forces conjuguées des armateurs, du gouvernement, mais aussi de la Seafarers International Union (AFL). Le cas de la CSU n'est pas unique ${ }^{51}$. On se rappellera qu'à partir de 1946, la SIU (AFL), dirigée par son président Harry Lundeberg, enverra Hal Banks au Canada, en lui confiant tous les pouvoirs et moyens financiers pour mettre sur pied la SIU ${ }^{52}$. Ces dirigeants corrompus de la SIU collaboreront ouvertement avec les armateurs au nom de la «légitime chasse aux communistes». Hal Banks, un escroc notoire, de collusion avec le gouvernement libéral canadien de l'époque, réussira à éliminer le syndicat communiste canadien. Dans les années qui suivirent, l'histoire de cette relation fut plutôt embarrassante pour le parti libéral ${ }^{53}$.

Lorsqu'en 1946 Hal Banks se vit confier la responsabilité de développer la branche canadienne de la SIU, les marins canadiens s'étaient déjà syndiqués à $100 \%$ au sein de la CSU sur la côte Est et à $90 \%$ sur les Grands lacs. La SIU profite des arrêts de travail pour

51 Mobilisation, 4, no 4: 20: En 1947 et en 1948, les communistes perdent la direction des TUA, des IWA (travailleurs de la forêt) en Colombie britannique, des quelques locaux des métallos où ils étaient encore présents. Le grand syndicat des marins (Canadian Seamen's Union) est détruit quand les patrons décident de reconnaître le syndicat du tristement célèbre Hal Banks (Seafarers International Union) qui prend le contrôle des ports et des bateaux par le chantage, l'extorsion et la violence. De nombreux petits syndicats, dans l'alimentation, le vêtement, la chaussure, etc... bâtis au travers de luttes épiques et glorieuses par des militants communistes, sont détruits ou pris en charge par les bureaucrates envoyés de Washington ou de la haute hiérarchie des centrales syndicales. ... Les communistes restent implantés dans deux puissants syndicats: les Mine Mill, dans les mines de l'Ouest et du nord de l'Ontario et les UE, le syndicat des grandes industries de masse de produits électriques. SIU qu'en 1949.

52 Hal Banks ne fut nommé officiellement président du district canadien de la

53 Voir Burton, W. Ayre, Mr. Pearson and Canada's Revolution by Diplomacy (Wallace Press, Montreal 1966), 86 à 89. Hal Banks avait un lourd passé. Depuis 1930, il avait comparu trois fois en cour. Les délits criminels dont on l'avait accusé couvraient toute une gamme allant de la contrebande de cigarettes au meurtre. Il était alors membre de l'exécutif du Sailors Union of the Pacific (AFL). 
envoyer ses membres travailler sur les navires en grève. Qualifiés de «briseurs de grève» à maintes reprises par le Searchlight, les membres de la SIU acceptaient de travailler à $\$ 20.00$ de moins que ceux de la CSU ${ }^{54}$.

Au printemps 1947 se produit la brusque trahison de Pat Sullivan qui démissionne de son poste de président de la CSU et de secrétaire-trésorier du CMTC. Le 14 mars, il donne une conférence de presse pour dénoncer les communistes infiltrés à l'intérieur de son syndicat. Il déclare entre autres que la CSU est contrôlée par le Parti communiste canadien et que la National Maritime Union (NMU) des États-Unis finance la CSU dans le seul but d'en prendre la direction. Il sera expulsé des rangs de la CSU pour avoir trahi le mouvement ouvrier. Sullivan fonde un pseudo-syndicat rival, The Canadian Lake Seamen's Union (CLSU), dont l'existence sera contestée par la CSU. En novembre 1947, même la Commission fédérale des relations ouvrières déclarera que l'Union de Pat Sullivan n'est pas «bona fide» 55 .

En fait, Sullivan fournira des briseurs de grève aux compagnies qui lui en feront la demande. Le Searchlight démontre à plusieurs reprises la collusion existant entre les compagnies maritimes et ce «nouveau McMasters». Par exemple, Ned Hayes, ancien collaborateur de Sullivan dans son organisation d'escrocs, fera la preuve que les fonds ayant servi à fonder la CLSU et à payer les briseurs de grève en 1947 et 1948 étaient fournis par la Canada Steamship Lines. Dans sa déclaration d'avril 1948, il prouvera que Sullivan travaille alors en étroite collaboration avec l'inspecteur Harrison de la RCMP56.

En 1947 et 1948 la CSU dénoncera à maintes reprises les actions irresponsables des bandes de voyous armés, ramassés par Sullivan dans les tavernes de la rue St-Laurent à Montréal pour harceler les marins syndiqués. Le Searchlight dénonce ces actions qui ouvrent la voie au fascisme. La collusion entre la SIU et Sullivan pour détruire la CSU sera également démasquée par le nouveau président de la CSU, Harry Davis, à partir de septembre $1947^{57}$. Au printemps 1948 l'Union des marins canadiens (CSU) lance même un

\footnotetext{
54 Le Searchlight (4 septembre 1948): 8.

55 Le Searchlight (25 septembre 1947): 2; (18 mars 1948): 4-5.

56 Le Searchlight (8 avril 1948): 1.

57 Le Searchlight (25 septembre 1947): 2 ; 18 mars 1948): 4-5.
} 
appel aux syndicats catholiques pour qu'ils cessent tout appui à Sullivan:

La liaison entre les syndicats catholiques de Montréal et Sullivan a aussi été démasquée. Les dirigeants des syndicats montréalais travaillent la main dans la main avec Pat Sullivan contre l'UMC. Dans l'intérêt du mouvement ouvrier et des syndicats eux-mêmes, les chefs de la Confédération générale des travailleurs catholiques doivent ordonner l'exclusion de la CLSU de Sullivan du Conseil Central des Syndicats catholiques de Montréal. Autrement, ils pourront être accusés eux aussi d'appuyer des syndicats de compagnies. ${ }^{58}$

Les difficultés à obtenir des contrats de la part des compagnies des Grands lacs, en particulier la Canada Steamship Lines et la Cie Misener, se sont accentuées en 1947-1948. Dès l'été 1947, la CLSU congédiait des équipages avec l'aide de la police pour remplacer les marins syndiqués par les briseurs de grève de Sullivan. L'édition du 31 juillet 1947 du Searchlight invitait les marins à se préparer à la grève contre ces deux importantes compagnies. En septembre, elles acceptaient de signer, mais quelques semaines plus tard, elles réengageaient d'autres briseurs de grève, violant ainsi leur contrat.

Le Ministère du Travail nomma des inspecteurs qui constatèrent le fait, sans pour autant forcer les armateurs à respecter leur contrat. Fin novembre, un vote réaffirme la confiance des marins dans la CSU. Les compagnies se voient obligées d'accorder la semaine de 40 heures.

À l'ouverture de la navigation, au printemps 1948, les mêmes compagnies firent marche arrière et se montrèrent intransigeantes. En mars 1948 elles signaient un contrat avec Sullivan, refusant de négocier avec des «communistes». Le journal Searchlight fait état des appuis de plusieurs syndicats, du Congrès des métiers et du travail du Canada par son président, Percy Bengough, de l'Union des ouvriers de l'auto, de l'Union des ouvriers de l'électricité, de l'Union des ouvriers de l'acier, et de syndicats d'outre-mer, en particulier de l'Union des marins d'Angleterre, qui condamnent tous les agissements des armateurs.

La Commission Brockington-McNish, instituée en novembre 1948 par le gouvernement fédéral face au différend entre la CSU et les compagnies, reconfirme la CSU dans son rôle d'agent négocia-

$58 \quad$ Le Searchlight (8 avril 1948): 2 . 
teur exclusif. Mais le gouvernement refuse, par la suite, de mettre un terme aux pratiques antisyndicales illégales des compagnies. Le ministre Humphrey Mitchell s'en lave les mains. Les expulsions d'équipages syndiqués par des fiers-à bras se multiplient; des affrontements de plus en plus nombreux se produisent. Des boyaux à vapeur seront même employés pour chasser les marins sur les lignes de piquetage.

Le Searchlight du 6 mai 1948 révèle que 35 arrestations orit eu lieu jusqu'alors. Â la fin de mai 1948 , près de 90 marins sont emprisonnés en vertu de l'Acte maritime ${ }^{59}$. Le 25 novembre 1948, 54 hommes seront encore au cachot. Tout le mouvement ouvrier est mobilisé. Les appuis à la CSU se multiplient. En août 1948, le Congrès des métiers et du travail du Canada convoque une conférence ouvrière d'urgence, regroupant des représentants de presque toutes les organisations du pays. À cette conférence, le viceprésident canadien de Brotherhood of Railway and Steamship's Clerks (AFL-TLC) essaie de semer la division par ses propos réactionnaires et en voulant faire porter la discussion sur la menace du communisme. Selon la CSU, Hall serait responsable de la collusion entre la SIU et la CLSU de Sullivan. Le Congrès des métiers et du travail du Canada continue à appuyer la CSU, malgré l'opposition de Hall ${ }^{60}$.

En septembre 1948, la suspension de Hall des rangs du CMTC posa le problème de l'autonomie de cette centrale face à l'AFL. Frank Hall, de concert avec l'AFL, avait saboté les efforts du CMTC et de la CSU pour éliminer l'organisation de Sullivan et la SIU. Hall avait même formé la Canadian Association of International Union Representatives, organisation ouvertement anticommuniste qui s'en prenait à la direction du CMTC. La suspension de Hall des rangs du CMTC fut approuvée à la quasi-unanimité des membres du Congrès. Mais l'AFL allait-elle endosser cette prise de position de la centrale canadienne qui lui était affiliée? La constitution du CMTC avait été amendée de façon à ce qu'il conserve pleine autorité sur les syndicats canadiens et éviter ainsi qu'un syndicat affilié à l'AFL puisse lui passer par-dessus la tête. L'AFL dut s'incliner temporairement devant l'affirmatif «Cooperation Yes! Domi-

59 Le Searchlight (20 mai 1948): 4.

60 Voir «L'Union des marins vs Frank Hall», article paru dans le Trades and Labor Congress Journal, 27 (septembre 1948): 63-64. 
nation No!» prononcé par le président du $\mathrm{CMTC}^{61}$. Hall sera rapidement réintégré au CMTC et préparera le terrain afin d'exclure la CSU du CMTC en faisant modifier les règlements ayant trait à la représentation en faveur des syndicats réactionnaires.

À la convention du CMTC de 1949, l'AFL réussira, en utilisant ses présidents canadiens des Unions internationales affiliées, à exiger l'expulsion de la CSU des rangs du CMTC ${ }^{62}$.

Voici comment le Trade and Labor Congress Journal (section française) ${ }^{63}$ présentant la version de l'exécutif du CMTC, explique la suspension de l'Union des marins, le 3 juin 1949. L'exécutif du CMTC reproche à la CSU d'avoir déclenché une autre grève sur les navires opérant sur la côte Atlantique, malgré le désaccord de l'exécutif du CMTC:

Depuis ce temps, la situation n'a pas cessé de se détériorer, elle se développa au point qu'elle intervint dans le bien-être des membres d'autres unions affiliées. Des lignes de piquetage qui, ostensiblement, étaient établies pour empêcher que d'autres viennent remplir les positions des marins en grève, dégénérèrent au point qu'elles empêchèrent des membres d'autres unions affiliées de remplir leurs occupations habituelles en violation de leurs ententes de travail. Ceci, à son tour, a développé des situations qui ne peuvent pas continuer, ni s'excuser.

Comme résultat ceci a engendré des dissensions internes dans le Congrès des Métiers et du Travail du Canada, au point que quatorze unions internationales affiliées ont pris la décision qu'elles ne continueraient pas leur affiliation aussi longtemps que l'Union des marins canadiens resterait affiliée.

Toutefois, lorsque les libertés que nous chérissons tant dans une constitution démocratique, sont si habilement employées pour mousser la cause communiste, il est alors plus que temps que des mesures de protection soient prises. ${ }^{64}$

Voyons dans quelles circonstances cette grève avait été déclenchée. En mars 1949, la Sharned and Colonial Steamship Ltd avait signé une entente avec la SIU. Harry Davis, président de la

61 Charles Lipton, op. cit., 276-279.

62 Charles Lipton, op. cit., 280-284.

63 «L'Union des marins canadiens suspendue», article paru dans Trades and Labor Congress Journal, 28 (1949): 36-37. Article traduit par Gustave Francq.

64 "Où en est la grève des marins? », dans Trades and Labor Congress Journal, 28: 33 . 
CSU, annonce alors qu'il faut prendre des procédures légales contre cette compagnie. Les membres des 3 navires d'une autre compagnie, ancrés dans le port de Halifax, prennent alors un vote de grève pour protester contre les agissements similaires de leur Compagnie. ${ }^{65}$ Le 31 mars, des représentants de la CSU négocient à Ottawa avec des représentants du Ministère du Travail pour que soient renouvelés les contrats ayant pris fin l'automne précédent. Au moment où le représentant du Ministère va annoncer à la Compagnie l'entente intervenue, les armateurs annoncent qu'entre-temps un accord a eu lieu avec la SIU. Harry Davis annonce alors la grève générale pour protester contre la provocation de la part des compagnies qui n'avaient cessé de signer des ententes avec la SIU, et ce au mépris de la loi que le gouvernement refuse de faire respecter.

Dès le déclenchement de la grève, le ler avril 1949, qui implique 90 navires et 3,000 marins, les compagnies obtiennent des injonction pour obliger les équipages à abandonner leurs navires. La tactique des compagnies est alors de remplacer les grévistes expulsés des navires par les tribunaux par des équipages de la SIU et de faire lever l'ancre pour les soustraire à l'action des piquets de grève.

La violence entre les membres de la USC d'une part et les gens de la SIU et la police d'autre part sera présente durant tout le conflit qui s'étendit rapidement aux ports des Grands lacs et de la côte du Pacifique. ${ }^{66}$.

Dans plusieurs cas, les marins travaillant à bord de navires ancrés dans des ports étrangers prennent un vote de grève et débraient. Des briseurs de grève ont été envoyés par avion. Pour appuyer les armateurs, le gouvernement fédéral annonce, le 17 mai 1949, que le «Navigation Act» oblige les marins à ramener leur navire dans un port canadien avant de se mettre en grève. Dans certains cas, le gouvernement canadien fait même appel à des autorités étrangères pour chasser les grévistes de leur navire et permettre aux briseurs de grève de ramener le navire au Canada ${ }^{67}$.

L'exécutif du CMTC accuse les dirigeants de la CSU d'avoir voulu causer du tort aux gouvernements travaillistes de Grande-

65 Le Devoir (22 mars 1949): 2. Voir à ce sujet, Pierre Bergevin, L'Union des marins canadiens et la grève de 1949 (1975), 28 p.

66 Voir en particulier Le Devoir (8 avril 1949): 3 qui fait état d'une bagarre qui fit une vingtaine de blessés à Halifax. La bagarre avait éclaté lorsqu'un train transportant 100 policiers et $\mathbf{2 0 0}$ membres du SIU arrivèrent au port d'Halifax.

67 Le Devoir (18 mai 1949): 3. 
Bretagne et d'Australie en paralysant leurs ports; on leur prête aussi d'autres intentions non-avouées:

La grève présentait une occasion des plus favorables d'importuner le gouvernement d'Angleterre, tout comme elle fournissait un medium effectif pour contrecarrer le plan Marshall et faire échec au Pacte de l'Atlantique. (...) La Fédération mondiale des trade-unions, comprenant les organisations entièrement contrôlées ou opérées par les gouvernements communistes, a maintenant pris la direction de cette grève. Ce qui a commencé comme une dispute industrielle bona fide est rendue maintenant bien loin des buts et aspirations des syndicats libres et ce fait doit être reconnu tout comme on doit y faire face. C'est une vaste campagne mondiale pour promouvoir la cause du communisme en réduisant, autant que faire se peut, l'efficacité du plan Marshall. ${ }^{68}$

L'exécutif dit avoir tenu compte dans sa décision d'exclure la CSU des pressions venant des Fédérations maritimes des autres pays:

Ceci comprend des organisations ouvrières d'Angleterre, de l'Australie, de la Nouvelle-Zélande, de l'Afrique du Sud et d'autres pays. Il est vrai de dire que ces organismes ont donné largement leur appui moral et financier à l'Union des marins au cours de leur grève. Mais si l'on en juge par les lettres que nous recevons elles rencontrent des difficultés toujours grandissantes avec le temps et sont désireuses maintenant de savoir combien longtemps encore durera cette grève.

L'exécutif du CMTC accuse l'exécutif de la CSU d'avoir produit «de la littérature plus ou moins grossière (...), insultant des membres de nos unions qui ont fait leurs preuves, qui ont même appuyé les marins et leur grève, mais ne peuvent se résoudre à endosser les actes de violence commis sans justification aucune dans leurs propres cités et districts ${ }^{69}$.

L'exécutif du CMTC avait même exigé la démission de certains membres de l'exécutif de la CSU afin de maintenir «de bonnes relations fraternelles au sein du Congrès ». Mais la CSU n'était pas prête à accéder à cette demande. Et l'exécutif de la CSU n'a pas accepté de mettre fin à la grève comme l'exigeait l'exécutif du CMTC. Le 3

68 «Où en est la grève des marins?» paru dans Trades and Labor Congress Journal, 28 (1949): 33. Article traduit par Gustave Francq.

69 "L'Union des marins canadiens suspendue", Trades and Labor Congress Journal, 28 (1949): 36-37. 
juin 1949 l'exécutif du CMTC annonçait la suspension de l'UMC des rangs du CMTC, suspension entérinée au Congrès du CMTC tenu à Calgary en septembre 1949.

Peter Newman, journaliste au Financial Post ${ }^{69}$, dans un grand reportage sur Hal Banks, résume ainsi cette grève de 1949:

Then came 1949 with more union war and bigger strike. "We'll strike the world!» bellowed Harry Davis ... And that piece of rhetoric about striking the world was only slightly swollen. More than a hundred Canadian ships were immobilized in four Canadian and many world ports. The strike spread to Britain and to other ports where Canadian ships were tied up - places like New Guinea and Africa, France, Norway and Australia, Marshall Aid Stuff for Europe was held up. London's docks were eventually paralysed. At the time, Britain's then Labor Minister, G.A. Isaacs said: «It was a bold and deliberate plan to restore the fortunes of the Communist-dominated Canadian Seamen's Union.»

But opposition to the C.S.U. because of its Communist leadership was becomming clamerous. The operators had their own assorted ressons for not liking it. The government and responsible opinion everywhere was appalled at having the critical shipping industry under Moscow management. Enthusiasm for getting another union into the picture was universal - except in Moscow. ${ }^{70}$

Voyons maintenant l'explication qu'en donne la CSU. La CSU essaiera de contrer la propagande bourgeoise en expliquant que ce ne sont pas les marins qui sont responsables de la grève mais bien les propriétaires de bateaux. Voici les faits: 1. Ce n'est pas une grève de juridiction, car tous les marins sont membres de l'Union des marins canadiens (CSU ou UMC), et la Seafarers International Union ramasse n'importe où des marins étrangers et des hommes qui n'ont jamais mis les pieds sur un bateau: "Nous défions les armateurs de produire un seul certificat de marin qualifié (A.B.) pour n'importe quel «scab» actuellement sur les bateaux», diront-ils.

2. C'est une grève pour les salaires, les conditions de travail et le garantie de l'emploi, même si la bourgeoisie et le CMTC y voient des «raisons politiques». Même si les armateurs niaient publiquement qu'ils voulaient couper les salaires, la CSU affirme: «Dans les négociations, les armateurs ont exigé des baisses de salai-

70 Peter Newman. "Hal Banks. White Knight on a Cadillac», The Financial Post (Toronto, October 16, 1954), no 42. 
res de \$20 à \$60. par mois.» Les conciliateurs ont recommandé 25 changements dans les conditions de travail établies depuis des années qui auraient pour résultat de couper les salaires nets des marins de $\$ 15$. à $\$ 20$, par mois. La Commission de conciliation a encore demandé l'abolition de l'engagement des marins par l'Union, ce qui aurait permis aux armateurs d'éliminer les navigateurs dévoués à l'Union et d'embaucher des gens sans expérience alors qu'il y avait déjà, suite aux congédiements des équipages canadiens, près de 1,000 marins canadiens sans travail. La CSU voulait que tous les vaisseaux naviguant sous pavillon canadien emploient des marins du Canada.

3. La CSU explique encore que le contrat des compagnies avec la SIU est illégal parce que la SIU ne représente pas les marins et qu'elle n'a aucun droit de négocier selon le Code fédéral du travail, l'Union des marins étant la seule union certifiée.

4. La grève est légale. Avant de déclarer la grève, l'Union des marins canadiens a passé par toutes les procédures exigées par les lois.

5. L'Union des marins a tout fait pour éviter la grève; elle explique que:

Le jour même où les armateurs ont forcé les marins à faire la grève, les chefs de l'Union discutaient avec les représentants du Ministère du Travail dans une autre tentative pour obtenir un règlement du différend avec les compagnies de navigation. Ils avaient réussi à obtenir une formule de règlement, quand ils furent soudainement avertis que les armateurs avaient signé un contrat illégal avec la SIU. Cette entente permet de renvoyer les membres de l'Union des Marins canadiens et établit une réduction des salaires nets des navigateurs. ${ }^{71}$

6. Les marins voulaient une augmentation de salaires et la sécurité d'emploi. La CSU démontre qu'une augmentation de $15 \%$ est logique quand le coût de la vie a monté de $17 \%$ depuis la signature du dernier contrat.

7. Enfin, la grève, selon la CSU, pourrait se terminer rapidement si seulement le gouvernement fédéral le voulait. Car une des principales compagnies responsables de cette grève, la Canadian National Steamships, appartient à un gouvernement; le gouvernement

71 Voir l'annonce du CSU parue dans La Patrie (23 avril 1949) : 45 qui résume les principaux arguments du CSU. 
pouvait forcer les armateurs et leur ordonner de cesser de violer les lois ouvrières. Il est évident maintenant que l'État canadien et la bourgeoisie canadienne avaient un même intérêt à écraser ce syndicat combatif et menaçant.

Lors de l'exclusion de la CSU l'exécutif du CMTC avait certaines réticences encore à affilier l'Union Internationale des Marins (SIU) :

[...] Le Comité a passé en revue avec une grande attention les circonstances dans lesquelles l'Union Internationale des Marins (Seafarers) est intervenue dans cette grève, l'empressement de ses membres et leur désir de servir sur des navires dont les hommes sont en grève et ce avec des équipages inexpérimentés et l'appui entier des compagnies intéressées, pour ne rien dire des méthodes répréhensibles qui furent suivies. De telles méthodes n'ont aucune place dans le mouvement syndical tel que nous le comprenons au Canada. Dans les circonstances le Conseil exécutif ne peut recommander l'affiliation de l'Union Internationale des Marins (SIU) au Congrès des Métiers et du Travail. ${ }^{72}$

En attendant, Hal Banks obtient des contrats avec diverses compagnies maritimes. Les armateurs, pour écarter la CSU, exigent des contrats comportant des baisses de salaires allant jusqu'à $30 \%$. Ils veulent ainsi maintenir la CSU en grève; ils avaient de cette façon le champ libre pour s'entendre avec Hal Banks. Les armateurs, avec la complicité du gouvernement, laissaient le conflit pourrir. Finalement, la SIU obtient vers 1950 le contrôle quasi total de la vie syndicale maritime au Canada. Au début des années cinquante, la SIU obtient son affiliation au CMTC. Hal Banks peut se construire un empire personnel par le terrorisme et la corruption. La situation est telle, qu'en 1960, le Congrès du Travail du Canada (CTC) expulsera la SIU de ses rangs en raison d'agressions contre un autre syndicat. Entre 1950 et 1960 , il n'y aura qu'une seule grève, soit en 1958 et elle ne durera que 8 jours! En février 1965, le syndicat international des Gens de mer (SIU) exigeait encore la semaine de 40 heures ${ }^{73}$.

\footnotetext{
72 «L'Union des marins canadiens suspendue», dans Trades and Labor Congress Journal, 28 (1949): 37.

73 La Presse (6 février 1965): 19. En 1949, la Canadian Seamen's Union avait 9,000 adhérents. En 1965, la Seafarers International Union regroupait 14,000 membres.
} 
Pendant les années troublées de la crise, de la guerre et de l'immédiat après-guerre, les dirigeants du syndicat des marins ont écrit une page particulièrement importante de l'histoire du syndicalisme canadien. Cette étude nous a permis de mettre en relief la polarisation inhérente à l'histoire du mouvement ouvrier en examinant le militantisme des travailleurs conscients et désireux d'améliorer leurs conditions de travail face à la collusion des forces bourgeoises - armateurs, gouvernement fédéral et dirigeants corrompus d'un syndicat international - en vue d'écraser un syndicat combatif. Nous avons mis en lumière le rôle déterminant des militants communistes dans l'essor du syndicalisme à cette époque.

Nous sommes conscients que cette étude, centrée sur le journal Searchlight, reste partielle. Il faudrait la compléter par l'examen systématique de nombreuses sources complémentaires. Certaines questions n'ont pas pu être développées dans le cadre de cette recherche. L'état général du mouvement syndical est à peine évoqué et les quelques éléments d'histoire du parti communiste que nous avons soulignés ne nous permettent pas d'apporter un éclairage suffisant sur toute une série de questions qui demeurent encore pour nous sans réponse. Comment évaluer la «collaboration tactique» des dirigeants de l'Union des marins canadiens avec le gouvernement et la quasi-absence de grèves chez les marins qui en a découlé, alors qu'au Québec le mouvement gréviste atteint un sommet en 19421943? Que sait-on de la résistance des travailleurs contre le gel des salaires imposé pendant la deuxième guerre? Les syndicalistes communistes ont-ils participé à cette riposte?

Ces limites ne sont que le résultat de l'état embryonnaire de la recherche sur l'histoire du mouvement ouvrier au Canada et au Québec. Elles sont, pour l'historien, une invitation à lancer de nouvelles enquêtes, à étudier d'autres cas qui permettront de mieux situer dans leur contexte des syndicats comme celui des marins. 